\title{
OFICINA TERAPÊUTICA COMO PROCESSO DE RESILIÊNCIA NO CENÁRIO DOS CUIDADOS PALIATIVOS E EXTENSIVOS
}

THERAPEUTIC WORKSHOP AS A RESILIENCE PROCESS CONCERNING A SCENARIO OF PALLIATIVE AND EXTENSIVE CARE

OFICINA TERAPÉUTICA COMO PROCESO DE CONTEXTO DE LOS CUIDADOS PALIATIVOS Y EXTENSIVOS

\author{
Paula Oliveira de Camargo* \\ Mariana Cunha Litholdo ${ }^{* *}$
}

\begin{abstract}
RESUMO
Cuidado paliativo é uma filosofia de abordagem diferenciada e integradora, cujo campo de atuação abrange doenças oncológicas e crônicodegenerativas. Cuidados extensivos é uma proposta de tratamento embasada nessa filosofia, que agrega a perspectiva de um processo de transição. $\mathrm{O}$ objetivo deste artigo é apresentar o processo de implantação das oficinas terapêuticas numa rede privada hospitalar, dentro dessa perspectiva. Será realizada uma descrição e análise de experiência desenvolvida cujo foco é a descrição das etapas do projeto desde sua implantação até o momento atual. Os benefícios identificados contemplam os seguintes fatores promotores de resiliência: o apoio social, autoconhecimento, resgate de sentido, formas de enfrentamento, questóes bioéticas e processo de luto. Conclui-se que as oficinas terapêuticas como modalidade de atendimento de grupo, de forma integradora e multiprofissional, é uma área de atuação nova bem como requer a necessidade de realização de pesquisa visando a contribuir para a ampliação de seus resultados.
\end{abstract}

Palavras-chave: Cuidados paliativos. Oficinas terapêuticas. Resiliência. Equipe multiprofissional.

\begin{abstract}
Palliative Care is an approach with a distinct and integrative viewpoint to oncological and chronic-degenerative diseases. Based on it, extensive care is a treatment proposition which aggregates the perspective of a transitional
\end{abstract}

\footnotetext{
Texto recebido em 17 de maio de 2017 e aprovado para publicação em 27 de agosto de 2018.

Mestra em Psicologia Clínica pela Pontifícia Universidade Católica do Rio de Janeiro (PUC-Rio), graduada em Psicologia pela PUC-Rio e em Administração de Empresas pela Pontifícia Universidade Católica do Rio Grande do Sul (PUC-RS), pósGraduanda em Psico-Oncologia e Formação em Cuidados Paliativos, tem o Certificado de Distinção de Conhecimento na Área de Psico-Oncologia concedido pela Sociedade Brasileira de Psico-Oncologia (SBPO), psicóloga hospitalar e profissional liberal. E-mail: p.camargo@placi.com.br.

* Terapeuta ocupacional graduada pela Universidade Federal do Triângulo Mineiro (UFTM), residência multiprofissional em Cuidados Paliativos pelo Instituto de Medicina Integral Prof. Fernandes Figueira (IMIP), experiência em contexto hospitalar e cuidados paliativos, residência multiprofissional hospitalar e redes de atenção à saúde pelo Hospital das Clínicas da Faculdade de Medicina da Universidade de São Paulo (HCFMUSP). E-mail: marilitholdo.to@gmail.com.

Afiliação Institucional: Hospital Placi - Cuidados Extensivos. Rua Dr. Martins Torres, 98 - Santa Rosa, Niterói-RJ, Brasil. CEP: 24240-705. Telefone: (21)2715-6330.E-mail: p.camargo@placi.com.br.
} 
process. The aim of this article is to present, within this perspective, the process of implementation of therapeutic workshops in a private hospital network. Description and analysis of activities performed, focusing on the stages of the project from its setup until the present moment, will be carried out. The identified benefits promote resilience and include factors such as social support, self-knowledge, recovery of a sense of meaning, coping, bioethical issues and the grieving process. Overall, it may be said that therapeutic workshops, as a modality of group care, are, in an integrative and multidisciplinary manner, a new field of work, which requires more research aiming to contribute to the expansion of its results.

Keywords: Palliative care. Therapeutic workshops. Resilience. Multidisciplinary team.

\section{RESUMEN}

El cuidado paliativo es una filosofía de enfoque diferenciada e integradora, cuyo campo de actuación abarca enfermedades oncológicas y crónicodegenerativas. Cuidados extensivos es una propuesta de tratamiento basada en esta filosofía, que agrega la perspectiva de un proceso de transición. El objetivo de este artículo es presentar el proceso de implantación de los talleres terapéuticos en una red hospitalaria privada dentro de esta perspectiva. Se realizará una descripción y análisis de experiencia desarrollada cuyo foco es la descripción de las etapas del proyecto desde su implantación hasta el momento actual. Los beneficios identificados contemplan los siguientes factores promotores de resiliencia: el apoyo social, el autoconocimiento, el rescate de sentido, formas de enfrentamiento, cuestiones bioéticas y proceso de duelo. Se concluye que los talleres terapéuticos como modalidad de atención de grupo, de forma integradora y multiprofesional, es un área de actuación nueva, así como requiere la necesidad de realización de investigaciones para contribuir a la ampliación de sus resultados.

Palabras clave: Cuidados paliativos. Talleres terapéuticos. Resiliencia. Equipo multiprofesional.

\section{INTRODUÇÃO}

Tuidado paliativo consiste numa proposta de abordagem diferenciada
e integradora, cujo campo de atuação abrange doenças oncológicas e
crônico-degenerativas. Com base no conceito de "dor total" delineado por
Cecily Saunders, na década de 1960, e legitimado no Brasil, em 2009, pelo
Conselho Federal de Medicina, por meio da Medicina Paliativa, atualmente vem
sendo implantado gradativamente no sistema de saúde, suscitando, no meio 
científico, opiniões controversas quando o assunto é relacionado ao paciente que não responde mais ao tratamento curativo.

No campo da prática clínica, designações como "fora de possibilidade de cura", "fora de possibilidade terapêutica de cura", "paciente terminal", entre outras expressões, reforçam a mentalidade médica tradicional de que "não há mais nada a se fazer", sugerindo uma possível descontinuidade na atenção e no cuidado por parte dos profissionais de saúde. De modo geral, pacientes portadores de doenças crônico-degenerativas ou em fase terminal são encaminhados em condições de extrema fragilidade física ocasionada pela progressão da doença.

Muitas vezes, as inúmeras tentativas em oferecer recursos terapêuticos que não promoverão benefício ao doente, compreendido na literatura médica como "tratamento fútil", restringem sensivelmente a possibilidade de uma atuação mais efetiva por parte da equipe de saúde no que diz respeito à oferta de cuidados. Tal circunstância indica que ainda prevalece uma mentalidade focada na cura e na intervenção como únicas modalidades de assistência, o que inviabiliza pensar na cura e no cuidado sob o ponto de vista da complementariedade para a condução de um tratamento.

Dessa forma, questôes pertinentes ao controle da dor, ao manejo de sintomas e aos cuidados no fim da vida ainda prevalecem como pontos cruciais para o encaminhamento do paciente para o cuidado paliativo, estabelecendo-se uma forte correlação entre a filosofia paliativa e os cuidados hospice, deixando a desejar quando o cuidado envolve situaçôes de maior complexidade, como no caso de doenças crônico-degenerativas.

Recentemente, no Brasil, cuidados paliativos têm sido um campo de trabalho que vem requerendo uma maior atenção na área de saúde. Mudanças nos padrões de expectativa de vida e no perfil epidemiológico das doenças, de acordo com Alves, Andrade, Melo, Cavalcante e Angelim (2015), são alguns dos desafios encontrados. A incidência de sobrecarga nos setores de saúde, devido à tanta fragilidade do sistema, quanto do cuidador familiar é outro ponto a ser analisado.

Delalibera, Presa, Barbosa e Leal (2015) salientam que, em situações de cuidado de fim de vida, quando a sobrecarga é demasiada, o cuidador familiar apresenta uma predisposição a desenvolver sintomas psicopatológicos e um luto complicado. Para Rojas e García-Vivar (2015), entre os vários temas que influenciam o retorno do paciente para casa, a sobrecarga do cuidado é um fator impactante na vida do cuidador familiar e, muitas vezes, pode requerer uma assistência direcionada e especializada. 
Nos anos de 2010 e 2015, a consultoria britânica Economist Intelligence Unit (2015), divulgou uma classificação sobre qualidade de morte em diversos países que ofereciam cuidados paliativos à sua população. Critérios avaliativos, como o ambiente de saúde e cuidados paliativos, formação do profissional, qualidade de cuidado e o engajamento da comunidade, contribuem para organização e colocação dos países, além de entrevistas e pesquisas com profissionais da área. O Brasil apresenta, respectivamente, as $38^{\mathrm{a}}$ e $42^{\mathrm{a}}$ posições.

A manutenção de seu baixo índice de qualidade de morte, segundo The Economist (Economist Intelligence Unit, 2015), e a existência de um modelo de transição de cuidados por meio do programa de atenção domiciliar são alguns dos fatores que evidenciam a necessidade de um campo de estudo mais aprofundado acerca do assunto. Nessas situações, as condutas paliativas introduzidas na hospitalização não se esgotam no processo de internação. Muitas vezes, tais condutas requerem dos cuidadores familiares a continuidade de atenção num cenário em que o paciente terá um percurso adaptativo em coerência com suas perspectivas prognósticas e seu processo de morrer. Nesse contexto, não se trata de paliar aquele que vai morrer em casa, mas sim cuidar daquele que, ao longo de seu processo de perdas cognitivo-emocionais e funcionais, construirá seu próprio caminho da morte.

Segundo a Organización Mundial de la Salud (2004), cuidado paliativo é a abordagem que promove qualidade de vida para pacientes e seus familiares mediante medidas de prevenção e alívio da dor, numa perspectiva multidimensional, desde o momento em que a doença é diagnosticada. Essa redefinição do conceito de cuidado paliativo, além de ampliar seu campo de atuação, corrobora a importância do papel da equipe multiprofissional na estruturação e manutenção de cuidados, principalmente em situações de progressão de doença prolongada sem possibilidade de cura.

Muito embora a filosofia dos cuidados paliativos contemple a possibilidade de uma concomitância entre cura e cuidado sob o ponto de vista de progressão da doença, o fato é que, na prática clínica, as situações de doenças crônicodegenerativas, muitas vezes, viabilizam um limiar muito tênue entre agravamento de sintomas e processo de morrer. Esse cenário, por sua vez, pode gerar sentimentos de angústia que transitam entre competências técnica e emocional, que podem interferir nos processos decisórios da equipe, inviabilizando a possibilidade de o paciente usufruir das potencialidades de um tratamento integrado.

$\mathrm{O}$ ingresso da equipe multiprofissional evidencia a mudança de paradigma, visto que a visão tradicional do modelo institucional cede lugar ao modelo sistêmico, cuja epistemologia incorpora os conceitos de multidisciplinaridade, 
interdisciplinaridade e transdisciplinaridade, na dimensão do cuidar. A importância destes consiste na busca da integração do conhecimento científico e da humanização junto à intervenção da medicina paliativa (Camargo, 2014).

A instituição hospitalar na qual o projeto foi implementado tem como seu foco de atuação o cuidado. Compreende este sob duas modalidades: a mudança do tipo de abordagem paliativa e a possibilidade de sua continuidade além do contexto de internação hospitalar, caracterizando uma intermediação entre a estruturação de um hospital de alta complexidade e home-care. Esse é um exemplo do conceito de hospital de transição com uma visão de cuidados extensivos.

Cuidados extensivos vêm a ser uma abordagem diferenciada, embasada na filosofia dos cuidados paliativos, aliada à perspectiva de um processo de transição, podendo ser este o retorno para domicílio. De acordo com Ferreira (2008, p. 786), a palavra transição provém do latim transit o e, entre seus significados, é definida como a passagem de um tratamento para outro ou o processo de mudança de uma condição para outra.

Os parâmetros para caracterizar um paciente com potencial de transição de cuidados se alicerçam na complexidade do tratamento e na severidade da enfermidade. Desse modo, pacientes com doenças crônico-degenerativas, sem possibilidade de cura, mas que requeiram cuidados contínuos até seu fechamento de ciclo vital, inserem-se nessa perspectiva.

Os tipos de cuidados são norteados pela condição diagnóstica em que o paciente chega à instituição. Constituem-se de 4 grupos distintos: cuidados paliativos oncológicos (CPO), cuidados paliativos não oncológicos (CPNO), cuidados continuados (CC) e cuidados de reabilitação (CR). Pertencem ao grupo de $\mathrm{CPO}$ aqueles usuários cujo diagnóstico clínico contemple doença de base neoplásica maligna, podendo, inclusive, apresentar modalidade de cuidados hospice. Já no grupo $\mathrm{CPNO}$, serão encontradas patologias de base neurológicas, pulmonares, cardiológicas e renais, em condição de cuidados paliativos de fim de vida.

As doenças neurológicas, pulmonares e renais com múltiplas comorbidades, com possibilidade de estabilização do quadro clínico com aporte de uma equipe multiprofissional, visam à reestruturação da atual realidade do paciente para que este possa retornar para seu domicílio, e são enquadradas no grupo de abordagem denominado CC. O que diferencia CPNO de CC no processo de transição vem a ser a mudança da condição clínica e, consequentemente, do tratamento paliativo. 
Também faz parte desse aporte de cuidados o grupo de reabilitação CR. De modo geral, o trabalho é focado na patologia de base e suas perspectivas de recuperação, sendo o processo de transição caracterizado pela possibilidade de uma maturação motora e cognitiva para a extensão dos cuidados em domicílio.

Logo, nos grupos $\mathrm{CPO}$ e CPNO, o processo de transição consiste na possibilidade do usuário e, ou, responsável decidir sob qual forma se deseja o fechamento do ciclo vital. Para os grupos CC e CR, trata-se de viabilizar ao usuário e, ou, responsável a preparação de todos os envolvidos para o retorno ao domicílio ou outro local que assista às necessidades para a continuidade do tratamento. A inserção da oficina terapêutica se delineou com base nessa segmentação dos grupos, considerando a existência de um cenário demarcado por experiências de enlutamento.

De acordo com Valladares, Lappann-Botti, Mello, Kantorski e Scatena (2003), oficina é um termo comumente utilizado para designação de atividades em saúde mental e requer atenção no que diz respeito a seu contexto conceitual, sob o ponto de vista de validação terapêutica. Em seu artigo, menciona formas de inserção dessa técnica no ambiente hospitalar, inicialmente dentro das instituições psiquiátricas, estabelecendo uma forte correlação entre o trabalho das oficinas como modalidade de entretenimento, ou seja, ação provida de prazer capaz de fazer o tempo passar, segundo afirma Saraceno (1999 apud Valladares et al., 2003).

Ocorre que tal metodologia provém de um modelo preconizado na década de 1940, dentro da rede pública de saúde, especificamente nos hospitais psiquiátricos, delimitando tal prática a um local de realização específico, com objetivos e características voltados a uma proposta de ocupação. Após a Reforma Psiquiátrica e, consequentemente, com a ruptura do ideário de confinamento, as oficinas requereram sua atenção, visto que passaram a ser reconhecidas como espaços facilitadores de comunicação e interação social, conforme pondera o autor.

Dessa forma, ampliaram suas modalidades de ação e passaram a ser legitimadas como espaço terapêutico capaz de promover transformação individual e coletiva. Vasconcelos (2000, apud Valladares et al., 2003) propõe o termo empowerment (empoderamento) como um novo olhar acerca dos objetivos do trabalho da oficina terapêutica, compreendendo esta como um "setting terapêutico isomórfico em relação à realidade externa (vida social e produtiva)" (Valladares et al., 2003).

Importante ressaltar que o objetivo do trabalho com oficinas terapêuticas vem sofrendo transformações. De acordo com o Ministério da Saúde, na Portaria SNAS no 189, de 19 de novembro de 1991, o atendimento em oficina 
terapêutica consiste em um dos procedimentos existentes na rede pública de saúde, com a finalidade de melhoria da qualidade de vida, diversificação de metodologia e técnicas terapêuticas, e a compatibilização entre as áreas de saúde mental com modelo assistencial para pacientes com transtornos psiquiátricos. Posteriormente, a OMS amplia o conceito de saúde mental, viabilizando pensar a prática da oficina terapêutica como um programa que contribui para a promoção da saúde mental, redução do estresse provocado pelo processo de adoecimento, valorização das capacidades cognitivas e, ou, funcionais atuantes e reinserção social.

Tal mudança de paradigma em relação aos trabalhos das oficinas viabiliza pensar a prática dessa metodologia em outro contexto hospitalar, com o foco voltado para resultados que priorizem o resgate da subjetividade, a reinserção social e a capacidade de potencializar os recursos físicos e psicológicos disponíveis como processo de resiliência.

Muito embora o termo resiliência seja oriundo das áreas da física e engenharia, cuja definição vem a ser a capacidade de um material para receber energia de deformação sem a sofrer permanentemente, para a área da saúde mental, trata-se de um conceito relativamente novo, passível de uma diversidade de constructos em se tratando de definição.

Para Walsh (2005), trata-se da capacidade de enfrentamento que um indivíduo tem diante da adversidade, retirando desta experiência recursos que o tornam mais fortalecido sob o ponto de vista biopsicossocial e espiritual. Para a autora, é a possibilidade que o sujeito tem de lidar com a situação de crise, transformando-a em perigo ou oportunidade de aprendizado, evocando formas de enfrentamento não adaptativas ou adaptativas, respectivamente.

Em artigo realizado por Taboada, Legal e Machado (2006), cujo objetivo foi investigar, em literatura especializada, os diversos conceitos de resiliência, os autores chamam a atenção para a necessidade de compreender que uma situação adversa pode promover várias formas de o indivíduo vir a desenvolver a resiliência. De acordo com os autores, a resiliência pode ser compreendida como um processo de adaptação versus superação, fator inato versus fator adquirido ou como algo circunstancial versus permanente.

Angst (2009), em revisão de literatura, salienta a incidência de variáveis que podem auxiliar, dificultar e favorecer a potencialização de recursos internos, denominados fatores de proteção, de risco e de eficácia, consequentemente. A autora afirma que pessoas resilientes apresentam características comuns relacionadas à resiliência, destacando que, de modo geral, tais atributos são 
facilitadores de adoção de estratégias de enfrentamento dotados de um sentido ou significado maior.

Em nossa análise e descrição de experiência desenvolvida, a observação do processo de resiliência consistiu na identificação do modo pelo qual o indivíduo lida com sua capacidade em ser resiliente dentro do contexto do adoecimento, envolvendo tanto o paciente quanto familiares e cuidadores. É necessário considerar que a experiência da doença abrange o modo como os pacientes, os membros da família e sua rede de suporte percebem, convivem e superam os desafios físicos e psicossociais dos sintomas dolorosos, da incapacidade e dos tratamentos, conforme pondera Walsh (2005).

O objetivo desse trabalho implementado éapresentar o processo de implantação das atividades de oficinas terapêuticas na rede privada: oficina de arte, oficina de música e oficina de cinema, e descrever sobre o espaço terapêutico em grupo como processo de resiliência.

\section{OBJETIVO}

Este estudo teve como objetivo geral integrar os conhecimentos teóricos da oficina terapêutica à prática dos cuidados extensivos existente na rede privada, planejando e implantando serviços que visem à promoção da estimulação e reabilitação de funções cognitivo-motoras e reintegração da saúde psicossocial.

Especificamente, os objetivos foram:

a) realizar avaliação dos aspectos psicoemocionais, atitudes e práticas dos participantes mediante estimulação proposta visando a contribuir com o diagnóstico diferencial;

b) desenvolver organização mental, criatividade, iniciativa e participação de acordo com a demanda;

c) enaltecer a comunicação verbal e não verbal entre os integrantes do grupo, visando a reforço positivo das potencialidades de cada um, viabilizando espaço de troca;

d) estimular aspectos cognitivos e habilidades motoras preservados;

e) trabalhar a sensação de bem-estar, potencializando a importância da qualidade de vida e integração do grupo;

f) estimular a interação entre os membros do grupo, propiciando a troca de informações a partir da compreensão singular de cada integrante. 


\section{MÉTODO}

\subsection{Local, participantes e período de realização das atividades}

As atividades de oficina terapêutica foram realizadas em um hospital da rede privada, no Município do Rio de Janeiro, tendo como participantes 58 pacientes adultos, sendo 53\% mulheres e 47\% homens, com faixa etária de 25 a 93 anos, e seus respectivos familiares e, ou, cuidadores.

O local escolhido para realização das atividades de oficinas de arte e música priorizou a área externa dessa instituição, um solário, com a finalidade de trabalhar um dos agentes estressores muito comuns em ambiente hospitalar: o confinamento. Na oficina de cinema, o espaço usado foi uma sala de uso comum do hospital, devidamente estruturado com equipamento audiovisual, justamente para estimular o trânsito do paciente dentro das dependências da instituição.

O perfil de pacientes atendidos é proveniente de operadoras de planos de saúde, tendo, em sua maior parte, escolaridade mínima de ensino médio. Em relação a ocupações profissionais, a maior parte do grupo encontra-se aposentada, sendo que aqueles que ainda mantinham atividade laboral pertenciam ao grupo de reabilitação. Em relação à predominância de tipos de doenças, as mais prementes nesse cenário foram doenças de base neurológica, cardiológica, renal, pulmonar, oncológica, além de suas comorbidades.

O serviço de Psicologia ficou responsável pela implantação e desenvolvimento inicial do projeto, sendo realizado em parceria com outros profissionais da equipe. Portanto, no decorrer do texto, serão mencionadas as contribuições do trabalho da Terapia Ocupacional para a estruturação e realização da atividade terapêutica.

A oficina terapêutica ocorre semanalmente, com modalidades intercaladas, e apresenta um caráter de grupo aberto, podendo o mesmo paciente participar no decorrer do período de internação.

Cada oficina é constituída por um número limitado de integrantes, podendo estar acompanhados ou não, e são obedecidos alguns critérios de inclusão e exclusão para a formação do grupo em coerência com a proposta. Para a oficina de arte, foram considerados fatores de inclusão pacientes que apresentavam funcionalidade motora preservada ou com perspectivas de reabilitação, além do funcionamento cognitivo preservado e fatores de exclusão, pacientes com ausência de funcionalidade motora. 
$\mathrm{Na}$ oficina de música, os fatores de inclusão considerados foram pacientes que apresentavam limitaçôes severas ou inexistentes de ordem motora, mas com condições de restabelecimento da parte oral, além da preservação das manifestações mentais. Os fatores de exclusão foram os pacientes sem qualquer capacidade fonoarticulatória.

Por último, na oficina de cinema, os fatores de inclusão considerados foram os pacientes com limitações fonoarticulatórias e motoras, mas com funções mentais, preservadas. Como fatores de exclusão, foram considerados pacientes com capacidade mental comprometida.

As atividades aqui descritas referem-se ao período de implantação do projeto ocorridas de agosto de 2015 a dezembro de 2016. Participaram 58 pacientes, sendo alguns acompanhados de familiar e, ou, cuidador. Em algumas situações, por exemplo, pacientes que iniciaram a atividade junto com o grupo e não conseguiram finalizar, foi concedido o material em uso para dar continuidade da atividade no leito.

\subsection{Materiais}

Os materiais utilizados foram: material escolar (tintas, pincéis, cartolinas, diversos tipos de papéis, tesoura, cola, lápis de cor, giz de cera, pincéis atômicos, barbantes, sucatas, telas de pintura, revistas, palitos de madeira, tecidos, fitas, enfeites, canudinhos, lantejoulas, etc.), rádio, CDs, caderno com as letras das músicas, material audiovisual e DVDs de shows musicais, documentários, peças de teatro e exibição de espetáculos.

\subsection{Procedimento}

O projeto das oficinas terapêuticas desenvolveu-se em momentos distintos, sendo a etapa inicial a oficina de música e cinema, no mês de março de 2015, e, posteriormente, a oficina de arte, no mês de julho do mesmo ano. Ambas as atividades terapêuticas contaram com grupos formados, em média, por seis integrantes, podendo estar acompanhados por familiares e, ou, cuidadores.

No primeiro momento, foram realizadas observações com base no planejamento inicial de cuidados delineado pela equipe multiprofissional. A Psicologia reavaliava, juntamente com a Terapia Ocupacional, as possibilidades de participação do paciente nas oficinas terapêuticas e qual modalidade se adequava melhor à proposta inicial de cuidados estabelecida pelas profissionais 
responsáveis, obedecendo aos critérios de inclusão e exclusão definidos para cada oficina.

Outro aspecto considerado era a escuta da equipe de Enfermagem em relação às manifestações psicossociais do paciente e sua família, decorrentes do processo de adaptação hospitalar. Após essa etapa e com base no levantamento das informações, era estabelecido para quais oficinas o paciente poderia ser convidado a participar.

Posteriormente, pelo serviço de Psicologia, foi realizada abordagem em leito, visando a uma complementação das informações já obtidas, mediante uma entrevista individual com paciente ou seu responsável, com o objetivo de dar informação sobre o trabalho da oficina terapêutica, identificar se era de seu desejo conhecer a proposta de trabalho.

A rotina se baseava, primeiramente, em verificar o prontuário eletrônico, obtendo-se informações sobre a condição clínica dos pacientes elegíveis para participar da oficina terapêutica. A seguir, era realizada uma seleção dos pacientes para a constituição do grupo do dia, repassando a informação à equipe de Enfermagem e aos encarregados de transporte. Nos casos de pacientes que não apresentavam mobilidade funcional, a Terapia Ocupacional participou, orientando quanto ao tipo de cadeira de rodas adequada para cada caso, realizando adequações necessárias.

Após essa avaliação dos pacientes com possibilidade de participação, era oferecido, pela equipe multiprofissional, o serviço da oficina terapêutica e identificava-se a disponibilidade e motivação desses pacientes para a atividade, sinalizando para a equipe de Enfermagem os seis pacientes constituintes do grupo do dia. Essa medida permitiu uma reorganização nas atribuiçóes da equipe de Enfermagem no que diz respeito à ordem de atendimentos da higiene matinal dos pacientes. Posteriormente, no caso de pacientes em treino de atividades de vida diárias (AVD), a terapia ocupacional acompanhou o processo de transferência leito-cadeira de rodas, concedendo as orientaçôes necessárias, visando ao estímulo da autonomia corporal do paciente.

As oficinas terapêuticas ocorreram uma vez por semana, com modalidades intercaladas, durante o período de aproximadamente uma hora. Todas as atividades desenvolvidas receberam supervisão dos profissionais envolvidos (psicólogo, terapeuta ocupacional e equipe de enfermagem) semanalmente. As atividades de arte e música, em sua maioria, foram realizadas ao ar livre, e as oficinas de cinema, em ambiente fechado. Ao fim das atividades que envolvem contato direto dos pacientes, todo o material de uso manual era higienizado, conforme as 
normas e orientações da Comissão de Controle de Infecção Hospitalar (CCIH), dando continuidade ao trabalho desenvolvido nas dependências do hospital.

\subsection{Resultados e Discussão}

No período de 2015 a 2016, foram realizadas 42 oficinas terapêuticas, contando com a participação de 423 pessoas, sendo $16 \%$ no ano de 2015 e $84 \%$ no ano de 2016, distribuídos em 211 pacientes, 102 familiares e 110 cuidadores. Diante da segmentação do grupo de cuidados que se estabeleceu a partir do ano de 2016, tivemos 3 pacientes do grupo CPO, 20 pacientes do grupo CR e 35 pacientes dos CC, totalizando 58 pacientes.

De acordo com as observações diretas realizadas em campo, foi possível identificar que as atividades oferecidas viabilizaram um espaço terapêutico em que os pacientes demonstraram sua capacidade de resiliência para lidar com a situação de adoecimento. Quiceno e Vinaccia (2011), em estudo baseado em evidência sobre resiliência, realizado em pacientes com enfermidade crônica em geral, incluindo o câncer, mencionam inúmeros fatores de proteção da saúde física e mental em situações desfavoráveis.

Já Bianchini e Dell'Aglio (2006) abordam o conceito de resiliência em situação de doença como a "capacidade de um indivíduo lidar com a doença, aceitando suas limitações, colaborando com a aderência ao tratamento, readaptando-se e sobrevivendo de forma positiva" (p. 430), compreendendo essa capacidade como um processo passível de ser desenvolvido, do ponto de vista subjetivo. Com base nessas colocações, serão analisados, neste estudo, os dados obtidos pelos pacientes na prática das oficinas, considerando os seguintes fatores promotores de resiliência: apoio social, autoconhecimento, resgate de sentido, formas de enfrentamento e questões bioéticas.

Ao longo dos encontros, foi detectada, entre seus participantes, a necessidade de trabalhar a função do apoio social do ponto de vista do real e do imaginário. Questóes como a receptividade à ajuda do outro, interação entre os membros, a importância da estimulação do outro por intermédio dos profissionais, pacientes ou cuidadores e atenção à escuta por meio dos feedbacks obtidos dentro do contexto grupal foram algumas das contribuiçóes obtidas para o resgate da função terapêutica do cuidar.

De acordo com Figueiredo (2012, p. 140), o cuidar é constituído de diversas fases que envolvem o exercício da função terapêutica por meio do ato de acolher, reconhecer, interpelar, identificar e respeitar o ritmo do outro, colocar-se disponível sem exceder o limite estabelecido na relação dual. Para Samea (2008, 
p. 88), o espaço grupal possibilita o contato e o reconhecimento do próprio fazer, seus limites e facilidades, a observação do fazer do outro, a percepção de semelhanças e contrastes, além da potencialização do fazer junto. Nesse sentido, as identificações e fantasias nos processos de interação conferem ao grupo um contínuo movimento, um efeito motor no sentido de provocar e potencializar transformaçōes.

Em relação ao autoconhecimento, compreendido por Quiceno e Vinaccia (2011) sob a perspectiva do reconhecimento de algo novo, foi identificada, entre seus integrantes, a obstinação para o alcance de um resultado que produzisse satisfação e bem-estar. Dessa forma, limitaçooes físicas e, ou, cognitivas não representaram impedimentos de realização, ocorrendo a percepção das próprias limitações e busca de alternativas para resolução de conflitos, autoquestionamentos sobre escolhas diferentes e obtenção de resultados favoráveis que legitimassem a expressão de suas preferências subjetivas, percepção de si mesmo como ser produtivo.

Durante os grupos, foram realizadas vivências corporais como consciência corporal e aprendizado do uso das capacidades funcionais, técnicas de posicionamento corporal, estratégias para redução da dor, espasticidade e outros possíveis fatores limitantes, potencialização das capacidades residuais e estimulação dos aspectos comprometidos. Além disso, foram utilizados dispositivos auxiliares, como engrossadores, e realizadas adaptações nos materiais quando necessário. $\mathrm{Na}$ experimentação e na realização de atividades que proporcionaram experiências corporais, ocorreu um resgate de aprendizado vivido corporalmente, possibilitando minimizar padrões posturais, criando possibilidades de uso do corpo, com o acompanhamento da Terapia Ocupacional (Antônio, \& Toldrá, 2005).

A possibilidade de elaboração de alguns limites e obstáculos, de forma coletiva, bem como a vivência concreta de potencialidades ofereceram aos pacientes a oportunidade de maior conhecimento e apropriação de si mesmos, de sua condição e da realidade. Um processo grupal pode sensibilizar cada sujeito em sua singularidade e provocar interessantes transformações em suas relações cotidianas; consigo mesmo, com os outros e com o mundo, conforme coloca Samea (2008, p. 89).

Como formas de enfrentamento, foram constatados aspectos positivos e negativos no decorrer das atividades, variando de acordo com a estimulação proposta ou com a intensidade de percepção ante as próprias limitaçóes. Determinação, persistência, motivação, maleabilidade com o momento possível e capacidade de tolerância mediante as limitações físicas existentes (dor postural, 
hemiplegias, etc.) sobressaíram-se em relação às atitudes de resistência em ser ajudado ou em realizar a tarefa em si. Outros pontos identificados foram a presença do senso crítico e do humor, a autoexigência na busca da perfeição de resultados justamente por imaginarem ser algo impossível de realização, num primeiro contato com o estímulo.

O trabalho da oficina terapêutica pode ser pensado como "a catalisação da construção de territórios existenciais" e a "reinvenção da vida em seus aspectos mais cotidianos", conforme pondera Valladares et al. (2003, p. 2). Tais comportamentos descritos acima, além de evidenciarem alguns dos resultados almejados, permitem refletir sobre a importância de disponibilizar-se, dentro da rotina hospitalar, um espaço de terapêutico de troca e elaboração, minimizando possíveis mecanismos de defesa como a negação, regressão e isolamento que repercutem de forma negativa em relação à doença, à internação e ao tratamento em si (Bianchini, \& Dell'Aglio, 2006).

Como resgate de sentido, entre alguns fatores que chamaram atenção ao longo das atividades, está a mobilização causada entre os participantes, do ponto de vista da imagem corporal. As oficinas, além de serem compreendidas como um espaço terapêutico, despertaram o senso de "encontro marcado", resgatando a autoestima de seus integrantes. Os pacientes passaram a cuidar de sua apresentação pessoal para ir ao encontro do outro, escolhendo com cuidado suas roupas, acessórios ou maquiagem. Aqui presenciamos o resgate do prazer em se cuidar, da reconquista da autonomia e capacidade de decisão, aspectos que, muitas vezes, perdem-se no processo de internação hospitalar.

A experiência do emprego de grupos pela Terapia Ocupacional tem demonstrado a exploração de possibilidades terapêuticas de diversas naturezas, salientando que os benefícios se estendem da elaboração do significado da experiência da incapacidade, conscientização dos limites, melhora das capacidades corporais e suporte emocional (Antônio \& Toldrá, 2015). Segundo Malta e Merhy (2010), no que diz respeito às doenças crônicas, é importante que a participação em grupos faça sentido e que propicie a criação de novos significados para o viver, como podemos perceber pela iniciativa dos pacientes no cuidado com a aparência, nas oficinas.

A possibilidade de se verem, por fotos ou vídeos, junto aos demais integrantes foi outro ponto relevante. Esse recurso, além de suscitar a troca de feedbacks entre os participantes, também viabilizou ao paciente dar-se conta de seu senso de estruturação e reorganização interna no que diz respeito à existência de seus próprios recursos e limitações, favorecendo a expressão de emoções como alegria, tristeza, insegurança, medo, entre outros compartilhados no contexto 
coletivo. Aqui podemos inferir que a oficina terapêutica pôde propiciar o restabelecimento da segurança física e, ou, psicológica, assegurando aos pacientes um espaço protegido no que diz respeito à aceitação de si mesmo, favorecendo um comportamento de apego adulto, que, conforme Sperling \& Berman (1994 apud Braz e Franco, 2017, p. 93), vem a ser a tendência em realizar "esforços importantes a fim de procurar manter proximidade e contato com uma pessoa ou pessoas específicas, que ofereçam potencial subjetivo para segurança física el ou psicológica”. Outro aspecto de fundamental importância, e que corrobora tal pensamento vem a ser a presença de rememoração de fases ou fatos da vida, compartilhado espontaneamente com o grupo.

Por último, importante salientar dois momentos distintos e significativos ocorridos no espaço da oficina, nos quais o grupo vivenciou situaçôes de perda e morte, sendo respectivamente, a despedida de uma profissional da equipe e o falecimento de um dos pacientes. Aqui, foram trabalhados terapeuticamente alguns dos princípios da bioética (respeito à autonomia e tomada de decisão) e o processo de luto pela legitimação de seus sentimentos, concedendo a cada integrante optar sobre sua permanência no grupo naquele momento.

Os pacientes, por confecção de cartôes, depoimentos e registros fotográficos, realizaram sua despedida da profissional da equipe. Esse momento propiciou experimentar o luto antecipatório dentro de uma unidade de cuidados, em que foi permitido "vivenciar a perda sem ela ter ocorrido efetivamente", conforme mencionam Braz e Franco (2017), viabilizando, de forma coletiva, um processo de ressignificação. Em relação à finitude de um paciente, o grupo conseguiu realizar suas escolhas, expondo seus sentimentos, sendo que uma parte optou pela continuidade do trabalho do grupo e outra pelo recolhimento a seus aposentos, evidenciando aspectos de proteção e de risco em relação à possibilidade de desenvolvimento de um luto complicado.

\section{CONCLUSÃO}

Os resultados mostraram que a oficina terapêutica pode promover o resgate e a ressignificação de alguns fatores que são perdidos no decorrer de uma internação prolongada, abrindo a possibilidade de trabalhar, de forma saudável, os fatores promotores de resiliência dentro do contexto hospitalar e promover a conscientização de alguns aspectos importantes para a adesão à proposta dos cuidados extensivos e paliativos.

Nos últimos anos, presume-se que, com a elevação da expectativa média de vida, o índice populacional de idosos crescerá proporcionalmente, com o 
aumento na incidência de doenças crônico-degenerativas. Tal acontecimento exigirá do sistema de saúde preparação e capacitação de profissionais para lidarem com situações limítrofes.

Cabe ressaltar que nem sempre essas situaçôes implicam necessariamente em finitude humana, mas sim numa rede de suporte assistencial que coadune tempo de sobrevida com qualidade e dignidade, envolvendo todos aqueles que propiciam esse cuidar, numa perspectiva de cuidados de transição.

A implantação desse projeto mostrou que as oficinas terapêuticas como modalidade de atendimento de grupo, de forma integradora e multiprofissional na rede privada, são um campo de oportunidades. Conforme os pacientes foram aderindo à proposta dos cuidados oferecidas pelo tratamento, legitimando a importância de estarem presentes a cada encontro, sugerindo ideias de melhorias nas atividades apresentadas, constatamos alguns dos componentes primordiais para a realização da filosofia paliativa: qualidade, valor e sentido de vida, conforme pondera Pessini (2008).

Atualmente, as oficinas vêm recebendo outras formas de estimulação, justamente por verificar demandas apresentadas pelos próprios pacientes, familiares e cuidadores. Um exemplo disso é a oficina de música, que, além de trabalhar com estímulo visual e verbal, incluiu os instrumentos musicais com o objetivo de ampliar as possibilidades de estimulação sensitivo-motora e percepto-cognitiva.

Quando o cuidado paliativo é concebido como forma de tratamento complementar à abordagem terapêutica, a aceitação do processo de finitude se configura como um novo panorama a ser desvendado de forma integrada, envolvendo paciente, familiares, cuidadores e equipe de saúde. Conclui-se que as oficinas terapêuticas como modalidade de atendimento de grupo, de forma integradora e multiprofissional na rede privada, podem se constituir numa área nova de atuação. Para isso, será certamente necessário dar continuidade a pesquisas que visem a contribuir para a discussão dessa técnica no contexto hospitalar de rede privada, e a possível ampliação de seus resultados, considerando os recursos de enfrentamento no adoecer, e na estimulação de componentes de trabalho ocupacional dentro do contexto dos cuidados paliativos. Talvez esse movimento seja o próximo passo para a compreensão de que aliar cura e cuidado numa perspectiva de complementaridade pode contribuir para adoção medidas preventivas que permitam aos membros da equipe de saúde trabalhar o objeto mais precioso desta teia relacional: a resiliência no processo do adoecer. 


\section{REFERÊNCIAS}

Alves, R. F., Andrade, S. F. O., Melo, M. O., Cavalcante, K. B., \& Angelim, R. M. (2015). Cuidados paliativos: desafios para cuidadores e profissionais de saúde. Fractal: Revista de Psicologia, 27(2), 165-176. Recuperado a partir de https://dx.doi.org/10.1590/1984-0292/943

Angst, R. (2009, julho-setembro). Psicologia e resiliência: uma revisão de literatura. Psicologia Argumento, 27(58), 253-260. Recuperado a partir de https://periodicos.pucpr.br/index.php/psicologiaargumento/article/ view/20225/19509

Antônio, P. P., \& Toldrá, R. C. (2015). A estratégia grupal na reabilitação de pessoas com acidente vascular cerebral: reflexões sobre a prática da Terapia Ocupacional. Cadernos Brasileiros de Terapia Ocupacional, 23(1), 43-52. Recuperado a partir de http://dx.doi.org/10.4322\%2F0104-4931.ctoAO440

Bianchini, D.C.S., \&Dell'Aglio, D. D. (2006). Processosderesiliência no contexto de hospitalização: um estudo de caso. Paidéia, 16(35), 427-436. Recuperado a partir de https://dx.doi.org/10.1590/S0103-863X2006000300013

Braz, M. S., \& Franco, M. H. P. (2017). Profissionais paliativistas e suas contribuições na prevenção de luto complicado. Psicologia: Ciência e Profissão, 37(1), 90-105. Recuperado a partir de https://dx.doi.org/10.1590/19823703001702016

Camargo, P. O. (2014). A equipe de saúde e a proposta de cuidados paliativos: uma nova realidade. (Dissertação de Mestrado). Programa de Pós-Graduação em Psicologia Clínica, Pontifícia Universidade Católica do Rio de Janeiro, Rio de Janeiro.

Delalibera, M., Presa, J., Barbosa, A., \& Leal, I. (2015). Sobrecarga no cuidar e suas repercussões nos cuidadores de pacientes em fim de vida: revisão sistemática da literatura. Ciência \& Saúde Coletiva, 20(9), 2731-2747. Recuperado a partir de https://dx.doi.org/10.1590/1413-81232015209.09562014

Economist Intelligence Unit. (2015). The 2015 Quality of Death index: ranking palliative care across the world. London: Economist Intelligence Unit. Recuperado a partir de http://www.lienfoundation.org/sites/default/ files/2015\%20Quality\%20of\%20Death\%20Report.pdf

Ferreira, A. B. H. (2008). Miniaurélio: o minidicionário da língua portuguesa. (7a ed.). Curitiba: Positivo. 
Figueiredo, L. C. (2012). As diversas faces do cuidar: novos ensaios da psicanálise contemporânea. São Paulo: Escuta.

Malta, D. C., \& Merhy, E. E. (2010). O percurso da linha do cuidado sob a perspectiva das doenças crônicas não transmissíveis. Interface - Comunicação, Saúde, Educação, 14(34), 593-606. Recuperado a partir de https://dx.doi. org/10.1590/S1414-32832010005000010

Organización Mundial de la Salud. (2014, 19-24 maio). 67a Asamblea Mundialde la Salud. Genebra: OMS. Recuperado a partir dehttp://apps.who.int/gb/ebwha/ pdf_files/WHA67-REC1/A67_2014_REC1-sp.pdf?ua=1 \&ua=1 \#page=60

Pessini, L. (2008). Cuidados paliativos: perspectivas contemporâneas. Revista Brasileira de Cuidados Paliativos, 1(1), 25-29.

Portaria SNAS no 189, de 19 de novembro de 1991. (1991, 19 novembro). Aprova a inclusão de Grupos e Procedimentos da Tabela do SIH-SUS, na área de Saúde Mental (Hospitais Psiquiátricos). Brasília: Ministério da Saúde. Recuperado a partir de http://www.maringa.pr.gov.br/cisam/portaria189.pdf

Quiceno, J. M., \& Vinaccia, S. (2011). Resiliencia: una perspectiva desde la enfermedad crónica en población adulta. Pensamiento Psicológico, 9(17), 6982. Recuperado a partir de http://www.scielo.org.co/scielo.php?script=sci_ arttext\&pid=S1657-89612011000200007\&lng=en\&tlng=es.

Rojas, M. A., \& García-Vivar, C. (2015). The transition of palliative care from the hospital to the home: a narrative review of experiences of patients and family caretakers. Investigación y Educación en Enfermería, 33(3), 482-491. Recuperado a partir de https://dx.doi.org/10.17533/udea.iee.v33n3a12

Samea, M. (2008). O dispositivo grupal como intervenção em reabilitação: reflexôes a partir da prática em Terapia Ocupacional. Revista de Terapia Ocupacional da Universidade de São Paulo, 19(2), 85-90. Recuperado a partir de http://dx.doi.org/10.11606/issn.2238-6149.v19i2p85-90

Taboada, N. G., Legal, E. J., \& Machado, N. (2006, dezembro). Resiliência: em busca de um conceito. Revista Brasileira de Crescimento e Desenvolvimento Humano, 16(3), 104-113. Recuperado a partir de http://pepsic.bvsalud.org/ scielo.php?script=sci_arttext\&pid=S0104-12822006000300012\&lng=pt\&nr $\mathrm{m}=\mathrm{iso}$

Valladares, A., Lappann-Botti, N., Mello, R., Kantorski, L., \& Scatena, M. (2003). Reabilitação psicossocial através das oficinas terapêuticas e/ou 
cooperativas sociais. Revista Eletrônica de Enfermagem, 5(1). Recuperado a partir de https://revistas.ufg.br/fen/article/view/768/850

Walsh, F. (2005). Fortalecendo a resiliência familiar. M. F. Lopes (Trad.), C. Bruscagin (Rev. Científica). São Paulo: Roca. 